(mothers during pregnancy; children both pre-alcohol use around ages 7-10, and post-alcohol use around ages 13-23).

Results The PRS were associated with multiple alcohol consumption phenotypes (strongest signal for alcohol amount at 18 weeks gestation: $p=1.01 \times 10^{-5}$ ) in pregnant mothers. There was an effect of maternal PRS for alcohol use on mother's perinatal depression $(p=0.02)$, offspring intellect $(p=0.016)$, and $\operatorname{ADHD}(p=0.04)$.

Discussion The effects of alcohol PRS previously found in the general population are also shown during pregnancy. We found an intrauterine effect of alcohol PRS on offspring intellect and ADHD. The effects shown are not due to offspring's own alcohol use, as these effects were not found within the child's analyses.

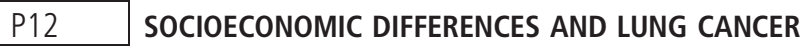 SURVIVAL IN GERMANY: DIFFERENCES IN CANCER THERAPY AND CLINICAL PROGNOSTIC FACTORS}

\begin{abstract}
${ }^{1,2}$ I Finke*, ${ }^{1} \mathrm{G}$ Behrens, ${ }^{3} \mathrm{~L}$ Schwettmann, ${ }^{4} \mathrm{M}$ Gerken, ${ }^{5} \mathrm{R}$ Pritzkuleit, ${ }^{6} \mathrm{~B}$ Holleczek, $1,7,8 \mathrm{H}$ Brenner, ${ }^{1} \mathrm{~L}$ Jansen. ${ }^{1}$ Division of Clinical Epidemiology and Aging Research, German Cancer Research Center (DKFZ), Heidelberg, Germany; ${ }^{2}$ Medical Faculty Heidelberg, University of Heidelberg, Heidelberg, Germany; ${ }^{3}$ Institute of Health Economics and Health Care Management, Helmholtz Zentrum München - German Research Center for Environmental Health, Neuherberg, Germany; ${ }^{4}$ Tumor Center- Institute for Quality Management and Health Services Research, University of Regensburg, Regensburg, Germany; ${ }^{5}$ Institute for Cancer Epidemiology at the University of Lübeck, Cancer Registry Schleswig-Holstein, Lübeck, Germany; ${ }^{6}$ Saarland Cancer Registry, Saarbrücken, Germany; ${ }^{7}$ Division of Preventive Oncology, German Cancer Research Center and National Center for Tumor Diseases, Heidelberg, Germany; ${ }^{8}$ German Cancer Consortium (DKTK), German Cancer Research Center (DKFZ), Heidelberg, Germany
\end{abstract}

\subsection{6/jech-2019-SSMabstracts. 163}

Background Studies from several countries reported lower lung cancer survival for lower socioeconomic status groups at individual and area level. Assumed reasons are differences in cancer care or tumor characteristics between these different groups. For Germany, this has not yet been investigated in detail. We examined the association between area-based socioeconomic deprivation and lung cancer survival by emphasizing on demographical factors related to the patient, clinical prognostic factors and utilization of cancer therapy.

Methods Patients registered with a primary tumor of the lung (ICD-10 C34) between 2000-2015 in three German population-based clinical cancer registries were eligible for our study. Area-based socioeconomic deprivation on municipality level was measured with the categorized German Index of Multiple Deprivation (GIMD). Our main outcome, survival after cancer diagnosis, was analyzed with Cox regression and we repeated the analysis for subgroups receiving chemotherapy, radiotherapy or surgery. Differences in stage and grading at diagnosis were analyzed with logistic regression. The main models included age, sex, histologic subtype, grading and stage at diagnosis. All analyses were conducted in SAS 9.4.

Results Overall, 22,905 patients were included of whom 72.9\% were male, $23.8 \%$ were over 75 years of age, $49.5 \%$ were diagnosed with stage IV cancer and $82.7 \%$ with non-small-cell lung cancer. Kaplan-Meier five year overall survival estimates from the least to the most deprived quintile were $17.2 \%$ [95\%-Confidence Interval (CI): 15.8-18.5], 15.9\% [14.8-17.2], $16.7 \%$ [15.5-17.9], 15.7\% [14.5-16.9], and 14.4\% [13.3-15.5], respectively. Our main Cox model showed lower survival in the most deprived group compared to the most affluent group
(Hazard Ratio (HR) 1.06, 95\%-CI 1.01-1.11). This association was more pronounced in the later than in the earlier period of diagnosis [2000-2010: HR 1.03, (0.98-1.09); 2011-2015: HR 1.12 , (1.03-1.23)]. No associations were seen for subgroups of patients receiving either chemotherapy, radiotherapy or surgery. When excluding patients diagnosed with stage IV, the effect estimate for the most deprived group was considerably larger [HR 1.15, 95\%-CI 1.07-1.24].

Conclusion Our preliminary results indicate differences in lung cancer survival according to area-based socioeconomic deprivation on municipality level in Germany.

\section{P13 INTRODUCTION OF CALORIE POSTING IN A UNIVERSITY TEACHING HOSPITAL STAFF CANTEEN: STAFF PERCEPTIONS AND RESULTING PURCHASING PATTERNS DIFFER ACCORDING TO GENDER}

${ }^{1} \mathrm{C}$ Flood, ${ }^{1} \mathrm{E}$ Cunniffe, ${ }^{1} \mathrm{~K}$ Doherty, ${ }^{1} \mathrm{~A}$ Lyons, ${ }^{1} \mathrm{~S}$ Stynes, ${ }^{2} \mathrm{~A}$ Pilkington, ${ }^{2} \mathrm{~L}$ Barnes, ${ }^{3} \mathrm{~T}$ Peare, ${ }^{1,4} \mathrm{C}$ Kelleher* ${ }^{1,4} \mathrm{P}$ Fitzpatrick. 'Department of Preventive Medicine and Health Promotion, St Vincent's University Hospital, Dublin, Ireland; '2Department of Dietetics, St Vincent's University Hospital, Dublin, Ireland; ${ }^{3}$ Department of Catering, St Vincent's University Hospital, Dublin, Ireland; "School of Public Health, Physiotherapy and Sports Science, University College Dublin, Dublin, Ireland

\subsection{6/jech-2019-SSMabstracts. 164}

Background Restaurants in Ireland will be required by law to display calorie counts on menus by end of 2019 as part of the strategy to combat obesity. Such initiatives are not always systematically evaluated. As part of the current National Healthy Ireland policy initiative, since September 2018 the introduction of calorie posting in hospitals is now Health Service Executive (HSE) policy also. The purpose of calorie posting is to promote awareness and increase consumption of healthier food and drink choices amongst HSE staff and the public using and visiting HSE healthcare facilities.

Methods The Health Promotion, Dietetics and Catering teams worked together to introduce both calorie posting and a traffic light system (TLS) for all foodstuffs and meals available in the main staff canteen in a large University teaching hospital. One month after the calorie posting launch day, a survey of staff members was undertaken over a working week-period (at 3 time points daily coinciding with main meals) to assess their perceptions. A short pre-designed Sphynx-software questionnaire was either interview or self-administered and left afterwards in drop-box. Analysis was conducted using SPSS.

Results In all 343 questionnaires were returned (65.3\% female; $18-44$ years $65.3 \%, 45+32.1 \%$, age unknown $2.6 \%)$. More staff found calorie posting helpful compared to TLS $(66.5 \%$ vs 43.7\%; $\mathrm{p}=0.001)$. More females than men found both calorie posting $(72.3 \%$ vs $53.3 \%$; $=0.001)$ and TLS $(49.1 \%$ vs $31.8 \%$; $\mathrm{p}=0.001$ ) helpful. $46 \%$ made themselves aware of calorie count always/mostly and $26.2 \%$ sometimes. More identified calorie posting as influencing food choice at least sometimes than TLS $(60.3 \%$ vs $36 \% ; \mathrm{p}=0.0001)$ Females were more likely to make a food choice change based on TLS (39.7 vs 20.6\%; p=0.01). Age and frequency of canteen use were not associated with either.

Conclusion The results suggest significant gender differences in that calorie posting is more used than the TLS and females are more likely to use both. Although self-selection may affect findings, nonetheless there were important moves in both genders and all ages to make healthier choices. Impact of price was not assessed. Health promotion strategies should account for gender preferences. 